LANCS-TH/9924

hep-ph/9912313

(December 1999)

\title{
Late-time creation of gravitinos from the vacuum
}

\author{
David H. Lyth \\ Department of Physics, \\ Lancaster University, \\ Lancaster LA1 $4 Y B$. U. K.
}

\begin{abstract}
Starting with the vacuum fluctuation, it is known that gravitinos will be created just after inflation, with number density $\sim 10^{-2} M^{3}$ where $M$ is the mass of the inflaton. Here, we argue that creation may be expected to continue, maintaining about the same number density, until a usually much later epoch. This epoch is either the 'intermediate epoch' when Hubble parameter falls below the gravitino mass, or the reheat epoch if that is earlier. We verify that such late-time creation indeed occurs if only a single chiral superfield is relevant, using the description of the helicity 1/2 gravitino provided recently by Kallosh et. al. (hep-th/9907124) and Giudice et. al. (hep-ph/9907510). Arguments are presented in favor of latetime creation in the general case. For the usual inflation models, $M$ is rather large and gravitinos from late-time creation are so abundant that a subsequent era of thermal inflation is needed to dilute them.
\end{abstract}

Introduction Gravitinos are created in the early Universe by thermal collisions [1], and some time ago [2] it was pointed out that they will also be created non-thermally, starting from the vacuum fluctuation that exists well before horizon exit during inflation. To see whether creation from the vacuum is significant, one needs equations which describe the evolution of the gravitino mode functions. Such equations have recently been presented [3, 田, for the case that only one chiral superfield is relevant. Using them, the authors find that the number density of gravitinos created just after inflation is of order $10^{-2} M^{3}$, where $M$ is the mass of the inflaton after inflation, and they have noted that these gravitinos may be more abundant after reheating than those created from thermal collisions. A similar result has been obtained for hybrid inflation, which involves at least two fields, assuming that in this context the gravitino becomes the goldstino of global supersymmetry [5] (see also [6]).

In this note, we argue that this is unlikely to be the end of the story [7]. Rather, creation is likely to continue, maintaining about the same number density, until either the 'intermediate epoch' when Hubble parameter falls below the gravitino mass, or the reheat epoch if that occurs earlier. We begin by verifying that such late-time creation 
indeed occurs if only a single chiral superfield is relevant, using the the description of the helicity $1 / 2$ gravitino provided recently in [3, 4]. Then we consider the case where other fields are relevant; a different field to break supersymmetry in the vacuum, a third field to allow hybrid inflation, and the fields corresponding to particles created just after inflation by preheating. In all these cases, we argue that late-time creation will continue until the intermediate epoch, unless it is terminated by preheating. Finally, we consider the cosmological consequences of late-time creation, which are rather dramatic in the most popular models of inflation.

Describing the gravitino in the early Universe To calculate the abundance of gravitinos created from the vacuum, one needs equations describing the evolution of the mode functions for the helicity $1 / 2$ and $3 / 2$ gravitino states, as seen by a comoving observer in the expanding Universe.

For the helicity $3 / 2$ state, one can safely proceed by using the Rarita-Schwinger equation with appropriate constraints, evaluated in Robertson-Walker spacetime, and with time-dependent mass $m_{3 / 2}(t)$ given by the usual $N=1$ supergravity formula. (We denote the vacuum value of $m_{3 / 2}(t)$ by $m_{3 / 2}$ without an argument.) A suitably-defined mode function satisfies the spin $1 / 2$ equation, with the same effective mass $m_{3 / 2}(t)$ that appears in the Rarita-Schwinger equation [8, 3, 4, 9]. As $m_{3 / 2}(t)$ can hardly be bigger than the Hubble parameter $H$ if it is to vary non-adiabatically, fhe abundance of helicity $3 / 2$ gravitinos created from the vacuum will no bigger than that of modulini created from the same mechanism, which is negligible [2, 10] compared with the abundance of gravitinos from thermal collisions.

For the helicity $1 / 2$ state, the same procedure may in general require modification, because the would-be goldstino (existing in the limit of global supersymmetry) may be a time-dependent mixture of the spin $1 / 2$ fields. To avoid this problem, the case where only a single chiral supermultiplet is relevant has been studied [3, 4]. It is found that a suitably-defined mode function again satisfies the spin $1 / 2$ equation, but with a different effective mass $\tilde{m}(t)$.

In a general model of inflation, the idealization of a single chiral supermultiplet will not be adequate. In hybrid inflation models one needs, in addition to the slowly rolling inflaton field, one or more additional fields to provide the constant part of the potential. Even if the inflaton field is the only one relevant for inflation, a second field is generally needed to break supersymmetry in the vacuum. Even so, the possibility that the inflaton field is the only relevant one is not excluded. Also, the field responsible for supersymmetry breaking may at some stage oscillate and dominate the energy density of the Universe, even if it is not responsible for inflation. Let us proceed on the assumption that only a

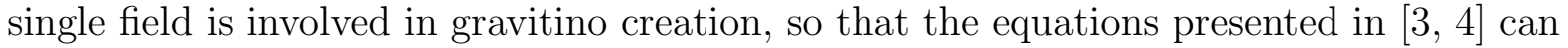
be used.

\footnotetext{
${ }^{1}$ The $N=1$ supergravity expression for $H^{2}$ contains a term $-m_{3 / 2}^{2}(t)$, which can hardly vary rapidly if it is canceled to high accuracy by the rest of the expression. Of course, a precise cancellation does occur at the present time, for some unknown reason (the cosmological constant problem).
} 


\section{Gravitino creation from the oscillation of the supersymmetry-breaking field} The effective mass $\tilde{m}(t)$ of the helicity $1 / 2$ mode function is given by [3, $\mathbf{t}^{2}$

$$
\tilde{m}(t)=m_{3 / 2}(t)-\frac{3}{2} m_{3 / 2}(t)\left(1+A_{1}\right)-\frac{3}{2} H A_{2}-\mu,
$$

where

$$
\begin{aligned}
A_{1} & \equiv \frac{P-3 M_{\mathrm{P}}^{2} m_{3 / 2}^{2}(t)}{\rho+3 M_{\mathrm{P}}^{2} m_{3 / 2}^{2}(t)} \\
A_{2} & \equiv \frac{2}{3} \frac{3 M_{\mathrm{P}} \dot{m}_{3 / 2}(t)}{\rho+3 M_{\mathrm{P}}^{2} m_{3 / 2}^{2}(t)} \\
A & \equiv A_{1}+i A_{2}=e^{i \chi} \\
\mu & \equiv \frac{1}{2} \dot{\chi} .
\end{aligned}
$$

In this expression, $M_{\mathrm{P}}=2.4 \times 10^{18} \mathrm{GeV}$ is the Planck scale.

The energy density $\rho$ and the pressure $P$ are supposed to be dominated by a real scalar field $\phi$. Taking its kinetic term to be canonical,

$$
\begin{aligned}
\rho & =V+\frac{1}{2} \dot{\phi}^{2} \\
P & =-V+\frac{1}{2} \dot{\phi}^{2},
\end{aligned}
$$

where $V(\phi)$ is the potential. The continuity equation is $\dot{\rho}=-3 H(\rho+P)$, equivalent to the field equation

$$
\ddot{\phi}+3 H \dot{\phi}+V^{\prime}=0
$$

where $H$ is the Hubble parameter, related to the energy density by $3 M_{\mathrm{P}}^{2} H^{2}=\rho$.

For future reference, let us note that the equations for $A_{1}, A_{2}$ and $\mu$ may be written in terms of $H, w \equiv P / \rho$, and $m_{3 / 2}(t)$, with the time-derivative of this last quantity eliminated using Eq. (3)). One finds

$$
A_{1} \equiv \frac{w H^{2}-m_{3 / 2}^{2}(t)}{H^{2}+m_{3 / 2}^{2}(t)}
$$

\footnotetext{
${ }^{2}$ In Eq. (3.23) of [3], the signs of the second and third terms should be reversed [11], after which that equation becomes identical with Eq. (1) upon making the identification $a^{-1} \Omega_{\mathrm{L}} \equiv-\tilde{m}(t)$. The sign of the effective mass is not physically significant, and in particular it will not affect the gravitino abundance. (Equivalently, the sign of the last term of Eq. 12) is not physically significant, since it corresponds to replacing a mode function by its complex conjugate.) We have checked [10] that Eq. (11) is equivalent to the more complicated expressions given in [4]. In both [3] and [4], it is assumed that the field is canonically normalized, but the results given there are valid also for arbitrary normalization [12. In fact, for a single real scalar field, one can always transform at least locally from arbitrary normalization to canonical normalization. In the supergravity model, $\phi$ is the real part of a complex field, and the corresponding transformation of the complex field is at least locally holomorphic, leading to an equivalent supergravity theory.
} 


$$
\begin{aligned}
A_{2} & \equiv \frac{\left[1-w^{2}+2(1+w) m_{3 / 2}^{2}(t) / H^{2}\right]^{\frac{1}{2}}}{1+m_{3 / 2}^{2}(t) / H^{2}} \\
\mu & \equiv \frac{3}{2} m_{3 / 2}(t)\left(1+A_{1}\right)-\frac{1}{2} \frac{\dot{w}-3 H(1+w)\left(w-A_{1}\right)}{A_{2}\left(1+m_{3 / 2}^{2}(t) / H^{2}\right)} .
\end{aligned}
$$

For momentum $k / a$, a suitably defined helicity $1 / 2$ mode function satisfies the equation

$$
u^{\prime \prime}+\left(k^{2}+(a \tilde{m})^{2}+i(a \tilde{m})^{\prime}\right) u=0,
$$

where the prime denotes differentiation with respect to conformal time $\mathrm{d} \eta=\mathrm{d} t / a$, and $a$ is the scale factor of the Universe such that $H=\dot{a} / a$. To calculate the gravitino abundance, one starts at early times with the negative-frequency solution $u \propto e^{-i \omega \eta}$, corresponding to the vacuum. At late times there is a linear combination of positive and negative frequency modes, and the occupation number is the coefficient of the positive frequency mode. Significant production occurs with momentum $k / a$ if there is appreciable violation of a weak adiabaticity condition [10

$$
\left|\overline{(a \tilde{m})^{\prime}}\right| \ll \omega^{2} \equiv k^{2}+(a \tilde{m})^{2},
$$

where the average is over a conformal time interval $\omega^{-1}$. In practice $k_{\text {max }}$, the biggest $k$ for which significant creation occurs, is simply the biggest value achieved by am, within the regime where $\tilde{m}$ varies non-adiabatically $\left(|\dot{\tilde{m}}| \gtrsim \tilde{m}^{2}\right)$.

Let us follow the evolution of $\tilde{m}$, to estimate $k_{\max }$. During inflation, $\tilde{m} \sim m_{3 / 2}(t)$ is much less than $H$ in magnitude. After inflation, $\phi$ oscillates about its vacuum value which we set equal to zero so that $\phi \simeq \phi_{0}(t) \sin M t$ where $M \gg H$ is the inflaton mass. We now have $\tilde{m} \simeq-\mu \equiv-\frac{1}{2} \dot{\chi}$, with

$$
\begin{aligned}
\cos \chi & \equiv \frac{\frac{1}{2} \dot{\phi}^{2}-V-3 M_{\mathrm{P}}^{2} m_{3 / 2}^{2}(t)}{\frac{1}{2} \dot{\phi}^{2}+V+3 M_{\mathrm{P}}^{2} m_{3 / 2}^{2}(t)} \\
& \simeq \frac{\cos 2 M t-m_{3 / 2}^{2}(t) / H^{2}}{1+m_{3 / 2}^{2}(t) / H^{2}} .
\end{aligned}
$$

When $\dot{\phi}=0, \cos \chi=-1$. The crucial point is that the maximum value of $\cos \chi$ is less than +1 , because $\cos \chi=1$ would correspond to $V=-3 M_{\mathrm{P}}^{2} m_{3 / 2}^{2}(t)<0$. It follows that $\chi$ oscillates in some range $\epsilon<\chi<2 \pi-\epsilon$. With the reasonable assumption $m_{3 / 2}^{2}(t) \sim m_{3 / 2}^{2}$, $\epsilon \sim m_{3 / 2} / H \ll 1$. While $\chi$ is rising, $\tilde{m} \simeq-M$, and while it is falling, $\tilde{m} \simeq+M$. Because of the sign switches, the evolution of the mode function is non-adiabatic, and gravitino creation continues, with every-increasing comoving momentum $k \sim a M$.

When $H$ becomes of order $m_{3 / 2}$, the sudden switches in sign of $\tilde{m}$ give way to a complicated variation, still on the timescale $M^{-1}$ and with typical value of order $M$. (If desired, this variation is conveniently calculated from Eqs. (9)-(11), with $w=\cos 2 M t$.) Creation finally ceases only when $H$ falls below $m_{3 / 2}$, and according to Eqs. (1) and (11) 
$\tilde{m}$ falls smoothly to the true value $m_{3 / 2}$, recovering the flat spacetime description of the gravitino.

We studied this example primarily to provide an existence proof, that late-time gravitino creation occurs in at least one case. This is the case that the oscillating field responsible for the energy density of the Universe, is the same as the field breaking supersymmetry in the vacuum. Before moving on, we note that this case may by realized in Nature if supersymmetry breaking is gravity mediated, though the mass $M$ would then probably be too small for late-time creation to be significant. Indeed, superstring theory suggests the existence of several fields (moduli) with only gravitational strength interactions, and mass very roughly of order $m_{3 / 2}$. (Kähler stabilization of the moduli can give masses several orders of magnitude bigger, for instance a dilaton mass of order $10^{6} \mathrm{GeV}$ is found in [13].) At least some of the moduli may well be displaced from the vacuum at the end of inflation, and one of them might be the inflaton). Any displaced modulus at first tracks the changing vacuum value, but it starts to oscillate at the intermediate epoch [14], and then dominates the energy density. Finally, one of the moduli is usually supposed to be responsible for supersymmetry breaking in the gravity-mediated case. The case that we have studied will be realized if this modulus is also the inflaton, and is the only one that oscillates. As far as the oscillation regime is concerned, the case is also realized if the inflaton is different from the supersymmetry breaking modulus, but decays early so that the latter is the only oscillating field at the intermediate epoch.

Late-time gravitino creation in the general case When additional fields and/or particles are involved, equations describing the evolution of the gravitino field are not yet available. However, late-time creation seems likely to occur quite generally up to the intermediate era, unless reheating occurs first.

Consider first models where the slowly-rolling inflaton field is the only one relevant for inflation, but something else breaks supersymmetry in the vacuum while ensuring that the potential vanishes there. In the model where the inflaton field did both jobs, late-time creation occurred because supergravity corrections to global supersymmetry become very important every time the potential the potential dips to zero, causing the mode function to vary non-adiabatically. One should not expect that the introduction of something else to break supersymmetry, would restore the adiabaticity.

Next, consider hybrid inflation models, where two fields are oscillating after inflation, with presumably a third breaking supersymmetry in the vacuum. In supersymmetric models, the two oscillating fields typically have the same mass $M$ after inflation. In general they are not oscillating in phase (though see [15] for a case where they are), which means that they cannot be replaced by a single field. Just after inflation, gravitino creation in this situation may be estimated in the global supersymmetry limit, as described in [5] for the case of $F$-term models. The number density will again be of order $M^{3}$, since the inflaton mass climbs to that value in a time of order $M^{-1}$. Again, there is no reason to expect gravitino production to stop, since supergravity corrections will again become important every time the potential dips to zero. 
In both of these situations, a definite calculation will clearly become possible when

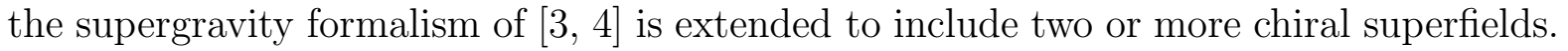
It is not so clear how to calculate things when particles as opposed to homogeneous fields become important, but still it is fairly clear what will happen. Consider first the case that preheating [16] converts most of the oscillating field energy into marginally relativistic particles. The energy of the latter will be somewhat reduced by redshifting even if it does not decay promptly into radiation, and one expects that after a few Hubble times oscillating fields again account for a non-negligible proportion of the energy density. Then late-time gravitino creation will presumably continue until the intermediate era, unless reheating happens first. After reheating, practically all of the energy density is in radiation, and one expects creation to stop, because nothing is varying rapidly. We emphasize that, although these expectations look quite reasonable, it is at the moment totally unclear how to describe the gravitino, when supersymmetry breaking comes mainly from the particle gas in the early Universe.

Cosmological significance of late-time gravitino production After production stops, the occupation number will be of order 1 below $k=k_{\max }$, giving number density 2]

$$
\begin{aligned}
n & \simeq \frac{2}{4 \pi^{2}} a^{-3} \int_{0}^{k_{\max }} k^{2} d k \\
& \sim 10^{-2}\left(k_{\max } / a\right)^{3} .
\end{aligned}
$$

The number density when creation stops is $n \sim 10^{-2} p^{3}$, where $p=k_{\max } / a$ is the maximum momentum of the created gravitinos.

We focus on the extreme case, where gravitino creation ends only at the intermediate era. This corresponds to energy density of order $M_{\mathrm{S}}^{4}$, where $M_{\mathrm{S}} \simeq \sqrt{M_{\mathrm{P}} m_{3 / 2}}$ is the scale of supersymmetry breaking. In gravity-mediated models of supersymmetry breaking, $m_{3 / 2} \sim 100 \mathrm{GeV}$ and $M_{\mathrm{S}} \sim 10^{10} \mathrm{GeV}$. In typical gauge-mediated models, $m_{3 / 2} \sim 100 \mathrm{keV}$ and $M_{\mathrm{S}} \sim 10^{7} \mathrm{GeV}$.

At the intermediate era, the gravitino number density is of order $10^{-2} M^{3}$. The relative abundance at nucleosynthesis is therefore [2]

$$
\begin{aligned}
\frac{n}{s} & \simeq 10^{-2} \frac{\gamma T_{\mathrm{R}} M^{3}}{M_{\mathrm{S}}^{4}} \\
& \simeq 10^{-2} \frac{\gamma T_{\mathrm{R}} M^{3}}{M_{\mathrm{P}}^{2} m_{3 / 2}^{2}}
\end{aligned}
$$

Here, $s$ is the entropy density at nucleosynthesis, and $\gamma^{-1}$ is the increase in entropy per comoving volume (if any), between reheating at temperature $T_{\mathrm{R}}$ and nucleosynthesis. If the entropy increase comes only from a late-decaying particle, there can be only a modest increase corresponding to $\gamma \sim T_{\mathrm{FR}} / T_{\mathrm{EQ}}$, where EQ is the epoch when the particle first dominates the energy density, and FR is the final reheat epoch when it decays. This gives 
$\gamma T \gtrsim T_{\mathrm{FR}} \gtrsim 10 \mathrm{MeV}$, where the bound comes from nucleosynthesis. However, $N e$-folds of thermal inflation [17, 14, 18] may also occur. This would reduce $\gamma$ by an additional factor $e^{-3 N}$. One bout of thermal inflation typically gives [14, 18] $N \simeq 10$ and a total $\gamma$ perhaps of order $10^{-15}$. Two bouts might give $N \simeq 20$ and up to $\gamma \sim 10^{-30}$ [14].

The cosmological significance [1] of the gravitino depends on its true mass $m_{3 / 2}$. With gravity-mediated supersymmetry breaking, one expects $m_{3 / 2} \sim 100 \mathrm{GeV}$ to $1 \mathrm{TeV}$, and observation then requires

$$
n / s \lesssim 10^{-13}
$$

The abundance of gravitinos from thermal collisions is then

$$
n / s \sim 10^{-13}\left(\gamma T_{\mathrm{R}} / 10^{9} \mathrm{GeV}\right),
$$

leading to the bound $\gamma T_{\mathrm{R}} \lesssim 10^{9} \mathrm{GeV}$. Using instead Eq. (19), we find

$$
10^{13} \frac{n}{s} \sim\left(\frac{M}{10^{7} \mathrm{GeV}}\right)^{3}\left(\frac{\gamma T_{\mathrm{R}}}{10^{9} \mathrm{GeV}}\right)\left(\frac{100 \mathrm{GeV}}{m_{3 / 2}}\right)^{2} \lesssim 1
$$

Gravitinos created from the vacuum are more abundant than those from thermal collisions, if $M \gtrsim 10^{7} \mathrm{GeV}$. Without thermal inflation, we need $\gamma T_{\mathrm{R}} \gtrsim 10 \mathrm{MeV}$ and therefore $M \lesssim 10^{11} \mathrm{GeV}$. In the worst case $T_{\mathrm{R}} \sim M_{\mathrm{S}}$, even a single bout of thermal inflation allows only $M \lesssim 10^{11} \mathrm{GeV}$. With two bouts, or with one bout and low $T_{\mathrm{R}}$, more or less any $M$ up to $M_{\mathrm{P}}$ might be accommodated.

With gauge-mediated supersymmetry breaking, one expects very roughly $1 \mathrm{keV} \lesssim$ $m_{3 / 2} \lesssim 100 \mathrm{GeV}$, with the upper decades disfavoured. Unless $m_{3 / 2} \gtrsim 100 \mathrm{MeV}$, this leads to a stable gravitino with present density

$$
\Omega_{3 / 2} \simeq 10^{5}\left(\frac{m_{3 / 2}}{100 \mathrm{keV}}\right) \frac{n}{s} \lesssim 1
$$

If creation from thermal collisions dominates, then very roughly [1]

$$
\Omega_{3 / 2} \sim\left(\frac{100 \mathrm{keV}}{m_{3 / 2}}\right)\left(\frac{\gamma T_{\mathrm{R}}}{10^{4} \mathrm{GeV}}\right) .
$$

Using instead Eq. (19),

$$
\Omega_{3 / 2} \sim\left(\frac{M}{10^{8} \mathrm{GeV}}\right)^{3}\left(\frac{100 \mathrm{keV}}{m_{3 / 2}}\right)\left(\frac{\gamma T_{\mathrm{R}}}{10^{4} \mathrm{GeV}}\right) \lesssim 1 .
$$

In this case, creation from the vacuum is more efficient if $M \gtrsim 10^{8} \mathrm{GeV}$, and we need thermal inflation if $M \lesssim 10^{10} \mathrm{GeV}$. In the favored case $m_{3 / 2} \sim 100 \mathrm{keV}$, corresponding to $M_{\mathrm{S}} \sim 10^{7} \mathrm{GeV}$, the constraints on $M$ are similar to those in the gravity-mediated case.

\footnotetext{
${ }^{3}$ We refer here to the thermal creation at the initial reheating. If thermal inflation subsequently occurs, the most significant thermal creation occurs at the final reheating, and $\gamma T_{\mathrm{R}}$ in Eq. (21) is to be replaced by the final reheat temperature.
} 
So much for the case that reheating happens after the intermediate epoch corresponding to $T_{\mathrm{R}} \lesssim M_{\mathrm{S}}$. By way of contrast, consider the opposite extreme of instant reheating, $T_{\mathrm{R}} \sim V^{1 / 4}$. Then,

$$
\frac{n}{s} \sim 10^{-2} \gamma\left(\frac{M}{V^{\frac{1}{4}}}\right)^{3} .
$$

Either $M / V^{1 / 4}$ should be sufficiently small, or thermal inflation is again needed.

Conclusion Let us end by considering the implications of late-time gravitino creation for models of the early Universe, and in particular of inflation. Supersymmetric models of inflation are reviewed for example in 119. In some of them, notably those using only flat directions, the mass of the field(s) oscillating after inflation is small, and late-time creation cannot be significant. However, in the most models, normalized to give the correct prediction for large scale structure, the inflaton mass is bigger than $10^{10} \mathrm{GeV}$. Using the popular names, examples include chaotic inflation, the most popular hybrid inflation models ( $D$ term, and the usual $F$ term model) and most new inflation potentials. The popular hybrid inflation models require $M \sim V^{1 / 4} \sim 10^{15} \mathrm{GeV}$ or so.

In all of these cases, the possibility of late-time gravitino creation drastically changes ones view about the reheat temperature. Starting the discussion with instant reheat, Eq. (26) represents a significant constraint. The constraint becomes stronger as the reheat temperature is lowered, because gravitino creation persists to a later epoch so that $n / s$ increases. Only when the reheat temperature falls below $M_{\mathrm{S}}$, does the constraint, now represented by Eqs. (22) and (25), start to become weaker. In many cases, the constraint cannot be met simply by lowering the reheat temperature. Instead, the entropy dilution factor $\gamma$ has to be small, often so small that thermal inflation is required.

To avoid these constraints, one might turn to models using only flat directions, leading to $M$ perhaps of order $m_{3 / 2}$, and giving negligible gravitino creation. Such models include the modular inflation mentioned earlier (so far without a concrete realization in the context of string theory) and certain hybrid inflation models.

The next step will be to check that late-time gravitino production actually occurs in the models mentioned, using equations that describe the gravitino in the presence of several fields. After that would come the more challenging task of describing the gravitino when a particle gas is the dominant source of supersymmetry breaking.

\section{Acknowledgments}

I am indebted to Andrei Linde, Renata Kallosh and Toni Riotto for useful discussions.

\section{References}

[1] T. Moroi, hep-ph/9503210; S. Sarkar, Rep. on Prog. in Phys. 59 (1996) 1493. 
[2] D. H. Lyth and D. Roberts, hep-ph/9609441, unpublished; D. H. Lyth, D. Roberts and M. Smith, Phys. Rev. D 57 (1998) 7120.

[3] R. Kallosh, L. Kofman, A. Linde and A. Van Proeyen, hep-th/9907124.

[4] G. F. Giudice, I. Tkachev and A. Riotto, JHEP 9908:009 (1999).

[5] G. F. Giudice, A. Riotto and I. Tkachev, hep-ph/9911302.

[6] A. L. Maroto and J. R. Pelaez, hep-ph/9912212.

[7] D. H. Lyth, hep-ph/9911257.

[8] A. L. Maroto and A. Mazumdar, hep-ph/9904206.

[9] M. Lemoine, hep-ph/9908333.

[10] D. H. Lyth, hep-ph/9909387 (v3), to appear in Phys. Lett. B.

[11] R. Kallosh, personal communication.

[12] R. Kallosh and A. Riotto, personal communications.

[13] M. K. Gaillard, D. H. Lyth and H. Murayama, Phys. Rev. D 58 (1998) 123505.

[14] D. H. Lyth and E. D. Stewart, Phys. Rev. D 53 (1996) 1784.

[15] M. Bastero-Gil, S.F. King and J. Sanderson, Phys. Rev. D 60 (1999) 103517.

[16] L. Kofman, A. Linde and A. A. Starobinsky, Phys. Rev. D 56 (1997) 3258.

[17] P. Binétruy and M. K. Gaillard, Phys. Rev. D 34 (1986) 3069; G. Lazarides and Q. Shafi, Nucl. Phys. B392 (1993) 61.

[18] T. Barreiro, E. J. Copeland, D. H. Lyth and T. Prokopec, Phys. Rev. D 57 (1998) 7345; T. Asaka, J. Hashiba, M. Kawasaki and T. Yanagida, Phys. Rev. D 58 (1998) 083509; T. Asaki and M. Kawasaki, hep-ph/9905467.

[19] D. H. Lyth and A. Riotto, Phys. Rep. 314 (1999) 146. 